\title{
The Microwave Spectrum of Excited Vibrational States of $\mathrm{CF}_{3} \mathrm{NC}$
}

\author{
Dines Christen* and Klaus-J. Ramme \\ Institut für Physikalische und Theoretische Chemie der Universität Tübingen \\ Z. Naturforsch. 39a, 865-870 (1984); received June 30, 1984

\begin{abstract}
The rotational spectra of vibrationally excited $\mathrm{CF}_{3} \mathrm{NC}$ states $\left(v_{4}, v_{6}, v_{7}, v_{8}, 2 v_{8}\right.$ and $\left.3 v_{8}\right)$ have been recorded and analyzed for the transitions $J: 1 \rightarrow 2,2 \rightarrow 3$ and $3 \rightarrow 4$ using (for the degenerate states) a simplified frequency expression based on perturbation theory. The following vibration/rotation interaction parameters were derived (in $\mathrm{MHz}$ ): $\alpha_{4}: 0.145, \alpha_{6}:-5.585, q_{6}^{+}$: $4.241, \alpha_{7}: 2.350, q_{7}^{+}: 1.438, \alpha_{8}:-10.610$, and $q_{8}^{+}: 5.101$. The Coriolis constant $\zeta_{88}^{\AA}$ was determined to be 0.672 in good agreement with the value calculated from the force field.
\end{abstract}

\section{Introduction}

In the course of analysing the rotational spectra of the vibrational ground state of $\mathrm{CF}_{3} \mathrm{NC}$ to determine the structure [1], two "anomalies" became apparent (see Figs. 1, 2, and 3):

1. In the highly resolved spectra of the ground state manifold, a few, albeit weak, lines could neither be explained as quadrupole hyperfine structure $(\chi: 1.06 \mathrm{MHz}$ ), nor as centrifugally induced $K$ structure $\left(D_{J K}: 8.33 \mathrm{kHz}\right)$.

2. To both sides of the ground state band, several further transitions were apparent.

These features were quickly identified as due to rotational transitions of vibrationally excited states, and the purpose of this report is to analyze these spectra.

\section{Experimental}

The sample was the same as used for the previous investigation [1]. Microwave spectra were recorded at $-40^{\circ} \mathrm{C}$ between 12 and $26 \mathrm{GHz}$ using a standard $20 \mathrm{kHz}$ Stark spectrometer.

\footnotetext{
* The assignment of $v_{2}$ and $v_{5}$ must probably be interchanged [3] but (as will be seen) this is not going to have a great effect on the vibration-rotation parameters discussed in this work.

Reprint requests to D. Christen, Institut für Physikalische und Theoretische Chemie der Universität Tübingen, Auf der Morgenstelle 8, D-7400 Tübingen 1.
}

\section{Rotational Spectra of Vibrationally Excited States}

The IR- and Raman spectra of $\mathrm{CF}_{3} \mathrm{NC}$ [2] show the following fundamentals (in $\mathrm{cm}^{-1}$ ):

$$
\begin{aligned}
& A: v_{1}: 2133, v_{2}: 1238^{*}, v_{3}: 837, v_{4}: 550, \text { and } \\
& E: v_{5}: 1219^{*}, v_{6}: 618, v_{7}: 458, v_{8}: 145 .
\end{aligned}
$$

At $-40{ }^{\circ} \mathrm{C}$ the Boltzman population of $v_{8}, 2 v_{8}$, $3 v_{8}, v_{7}, v_{6}$, and also $v_{4}$ enables a recording of their respective rotational spectra, whereby the $v_{8}$ progression appears as a strong series of bands to higher frequency of the ground state lines, whereas the much weaker $v_{7}$ and $v_{4}$ fundamental states lie to lower frequency, very close to the ground state. $v_{4}$, in fact, appears within the ground state band even at low $J$, and for higher $J, v_{7}$ is interfered by higher $K$-lines from the ground state. The $v_{6}$ state appears as a weak band between the ground state and the $v_{8}$ state.

Consequently the $v_{8}$-analysis ought to be quite consistent, whereas the analyses of the much weaker $v_{7-}, v_{6-}$, and $v_{4}$-states are more tentative.

It must be noted, however, that the analysis of all excited degenerate states suffers from the fact that only low- $J$ transitions have been observed, thus the somewhat clearer picture provided by higher $J$ (and $K l-1 \mid$ ) transitions could not be seen. For the same reason, the usual perturbation treatment of Amat, Nielsen and Tarrago [4] was expected to suffice for the calculations while usually no serious resonances appear in low- $J$ transitions; terms including $q_{12}^{\mathrm{t}}, d_{12}^{\mathrm{tt}}$ and $f_{24}^{\mathrm{t}}$ were therefore neglected all together. 


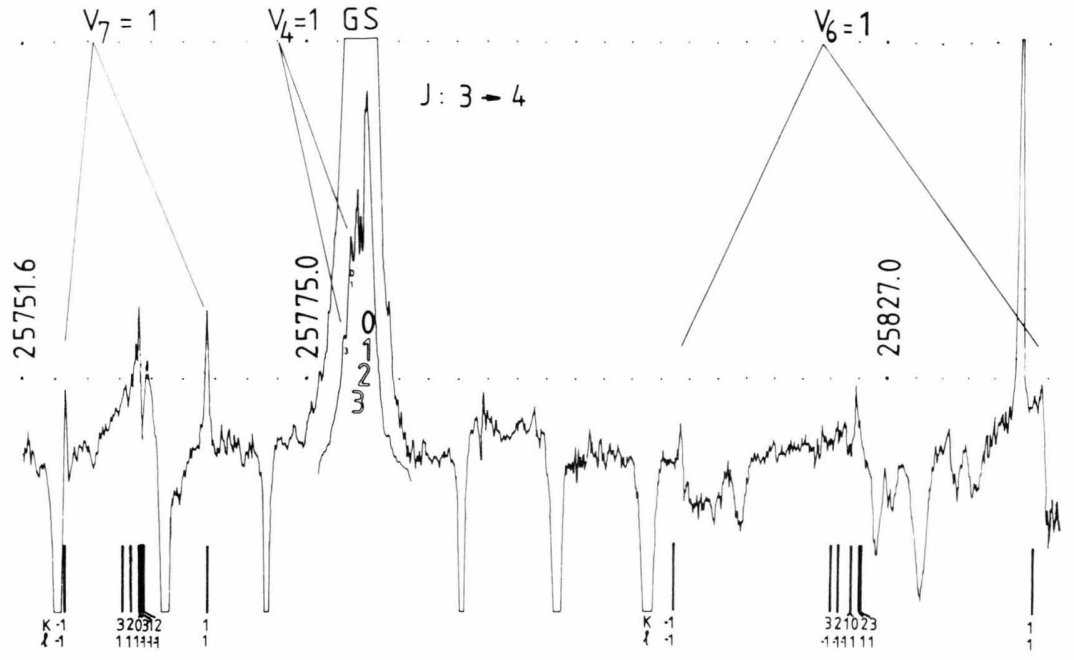

Fig. 1. An overview of the $J: 3 \rightarrow 4$ rotational transition at $25775 \mathrm{MHz}$, showing the ground state as well as the $v_{4^{-}}, v_{6^{-}}$, and $v_{7}$-excited vibrational states. Frequency markers (dots) are $2.6 \mathrm{MHz}$ apart. The calculated spectra for the excited degenerate states (using the constants of Table 2) have been indicated using a stick diagram, which also shows the assignment. Experimental conditions: Pressure: 7 mtorr, Stark field: $400 \mathrm{~V} / \mathrm{cm}$, time constant: $300 \mathrm{msec}$.

Table 1. Simplified Frequency Expressions for low- $J$ Transitions in the Ground-and Excited degenerate States $\left(d_{i}=2\right)$.

$$
\text { GS: } \quad(l=0): \quad F=0
$$

$$
\begin{aligned}
& v=2(J+1) B_{v}-4(J+1)^{3} D_{J}-2(J+1) K^{2} D_{J K}+2(J+1)(K l) \eta_{J}+F\left(v, q^{+}, Z, Y\right) \\
& B_{v}=B_{0}-\alpha_{i} v_{i}+\gamma_{i}\left(v_{i}^{2}+d_{i} v_{i}\right) *
\end{aligned}
$$

$v=1:(l= \pm 1): F= \pm q^{+}(J+1)$

$$
\begin{aligned}
& F=\frac{\left(q^{+}\right)^{2}}{4 Z}\left(\frac{(J+1)^{3}}{K l-1}-(J+1)(K l-1)\right) \\
& v=2:(l=0): \quad F=\frac{\left(q^{+}\right)^{2}}{K^{2} Z^{2}-(Z+Y)^{2}}\left((J+1)^{3}(Z+Y)+K^{2}(J+1)(Z-Y)-(J+1)(Z+Y)\right) \\
& (l= \pm 2): F=\frac{\left(q^{+}\right)^{2}}{4(K l Z-2(Z-Y))}\left(4(J+1)^{3}-(J+1)(K l-2)^{2}\right) \\
& F=2 F(K l \neq 4) \\
& F=0 \\
& \imath=3:(l= \pm 1): F= \pm 2(J+1) q^{+}-\frac{3\left(q^{+}\right)^{2}}{4((K l+1) Z+2 Y)}\left((J+1)^{3}-(K l+1)^{2}(J+1)\right) \\
& F=\frac{\left(q^{+}\right)^{2}}{(K l-1) Z}\left((J+1)^{3}-(K l-1)^{2}(J+1)\right)-\frac{3\left(q^{+}\right)^{2}}{4((K l+1) Z+2 Y)}\left((J+1)^{3}-(K l+1)^{2}(J+1)\right) \quad K l \neq 1 \\
& (l= \pm 3): F=\frac{\left(q^{+}\right)^{2}}{4(K l Z-3(Z-2 Y))}\left(9(J+1)^{3}-(K l-3)^{2}(J+1)\right) \\
& Z=A_{v^{v}}\left(1-\zeta_{v v}^{z}\right)-B_{v^{\prime}} ; \quad Y=x_{v v}-A_{v} \zeta_{v v}^{z} \\
& K l \neq 4 \\
& K l=4(+) \\
& K l=4(-) \\
& K l=1( \pm)
\end{aligned}
$$

* The $l$-dependence of $B_{r}$ for $v>1$ seems to be quite small in $\mathrm{CF}_{3} \mathrm{NC}$, and has been neglected in this expression.

With these simplifications, and using $q^{+}=4 q_{0}^{\mathrm{t}}$, $Z=A_{r}\left(1-\zeta_{v v}^{z}\right)-B_{v}$ and $Y=x_{v v}-A_{v} \zeta_{v v}^{z}$ with $x_{v v}$ the anharmonicity constant, the expressions for the ground and excited degenerate states can be written as shown in Table 1.

The advantage of these expressions is the often recurring combinations of parameters, which permits a semi-automatic calculation of these transitions on a programmable HP 97 desk top calculator.
The similarity between $\mathrm{CF}_{3} \mathrm{CN}$ and $\mathrm{CF}_{3} \mathrm{NC}$ concerning vibrational spectra $[2,5]$ and rotational constants $[1,6]$ led us to expect rotation-vibration interaction parameters for the degenerate states not very unlike those found in $\mathrm{CF}_{3} \mathrm{CN}[7,8]$, except that the numerical values especially for the $v_{8}$ parameters would be larger than those found in trifluoro acetonitrile, due to the weaker bonding in $\mathrm{CF}_{3} \mathrm{NC}$ [1]. 
$v_{8}$-Progression (see Figs. 2 and 3 )

The lowest frequency vibrational mode in $\mathrm{CF}_{3} \mathrm{NC}$ is the $\mathrm{C} \equiv \mathrm{N}-\mathrm{C}$ degenerate bending mode, $v_{8}$ at $145 \mathrm{~cm}^{-1}$ with a population relative to the ground state of $82 \%$ in the $v_{8}=1$ state, and $33 \%$, and $13 \%$ respectively in the $v_{8}=2$ and 3 states $\left(\right.$ at $-40^{\circ} \mathrm{C}$ ). The spectrum shows the usual pattern of a relatively compact central group of lines flanked by two equidistant single lines, the $l$-type doublets having $|K-l|=0$. The position of the central lines for these low- $J$ transitions are well described by perturbation theory, and model calculations quickly led to an assignment of the $J: 1 \rightarrow 2$ transitions with $|K-l|=1$ and 2 , since the obscuring quadrupole hyperfine structure of the $|K-l|=1$ line could be well accounted for using the quadrupole coupling constant $(\chi: 1.06 \mathrm{MHz})$ determined for the ground state [1] in a first order calculation.

With the derived preliminary parameters, the higher $J$ transitions were predicted and subsequently assigned. At the maximum $J(J=4)$ no conflicting evidence to perturbation theory was encountered.

The bootstrap method used in the $v_{8}=1$ state was now extended to establish an assignment for the $v_{8}=2$ state and subsequently for the $v_{8}=3$ state. In the last two states, two values of $l$ are possible, but both sets of transitions seem to be centered on the same frequency (with a maximum deviation of approximately $0.2 \mathrm{MHz}$ for the measured transitions), thus only one $\alpha$ (and $\gamma$ ) were needed to account for the frequency shifts of all excited $v_{8}$ states relative to the ground state.

As can be seen from Table 1, 9 parameters are thus needed in order to fit the observed spectrum. With so few measured transitions, stable solutions are hardly possible. To stabilize the normal equations for the least squares fit, the ground state transitions plus the transitions of all excited $v_{8}$ states were used simultaneously. The ground state lines with a bigger span of $J$ - and $K$-values - predominate in the determination of $B_{0}, D_{J}$ and $D_{J K}$, the $|K-l|=0$ lines of the first and third excited states more or less determine $q^{+}$(and partly $\alpha, \gamma$ and $\eta_{J}$, whereas $Z$ (and $\alpha, \gamma$ and $\eta_{J}$ ) are determined by the remaining lines. $Y$, and thus the anharmonicity, remains largely indeterminate. Again higher $J$ transitions with the enhanced possibility of resonances are needed in order to establish the value of $Y$. The obtained results are collected in Table 2.

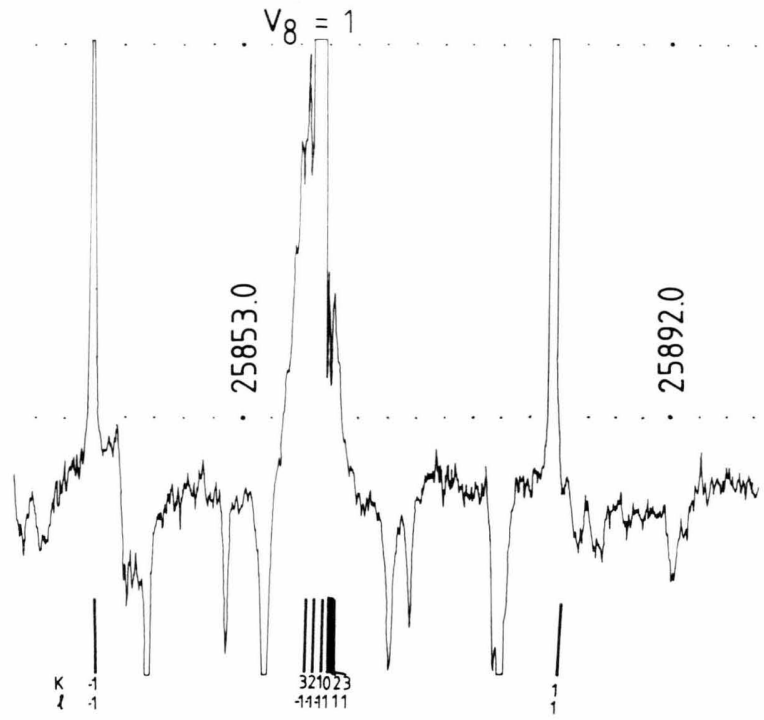

Fig. 2 . The $v_{8}=1$ excited vibrational state $(J: 3 \rightarrow 4)$ showing the $l$-type doublets as well as the central peak. Experimental conditions are as in Figure 1. Frequency markers 2.6 MHz apart.

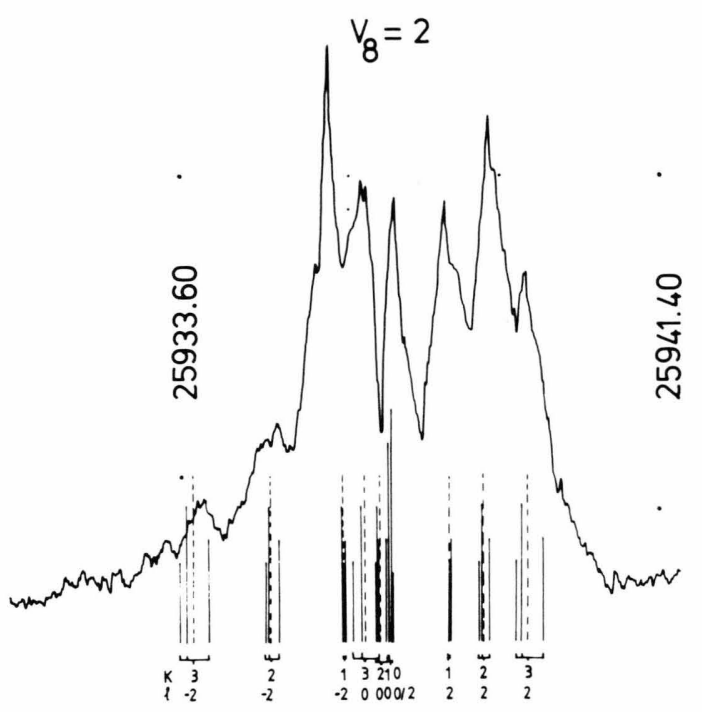

Fig. 3. The $v_{8}=2$ excited vibrational state $(J: 3 \rightarrow 4)$. This higher resolution spectrum is shown as a representative of the spectra that have actually been used for the determination of the line frequencies, whereas Figs. 1 and 2 gives in impression of the overall appearance of a rotational transition. Quadrupole hyperfine structure has been indicated using the following intensity scheme: $F: 4 \rightarrow 5$, $41 \%, F: 3 \rightarrow 4,31 \%$, and $F: 2 \rightarrow 3,24 \%$. Hypothetical center frequencies are indicated as broken lines. Frequency markers are $2.6 \mathrm{MHz}$ apart. Experimental conditions: Pressure: 5 mtorr, Stark field $400 \mathrm{~V} / \mathrm{cm}$, time constant: $1.25 \mathrm{sec}$. 
Table 2. Measured Transitions (meas.-calc.) in Excited Degenerate States of $\mathrm{CF}_{3} \mathrm{NC}$ with derived Rotational Constants ( $1 \sigma$ ). Units are MHz except for centrifugal distortion constants $(\mathrm{kHz})$ and $\zeta$ (Dimensionless).

\begin{tabular}{|c|c|c|c|c|c|c|c|c|c|}
\hline$J$ & $K$ & $*$ & $\begin{array}{l}v_{6} \\
|l|=1\end{array}$ & $\begin{array}{l}v_{7} \\
|l|=1\end{array}$ & $\begin{array}{l}v_{8} \\
\mid l=1\end{array}$ & $\begin{array}{l}2 v_{8} \\
l=0\end{array}$ & $\begin{array}{l}2 v_{8} \\
|l|=2\end{array}$ & $\begin{array}{l}3 v_{8} \\
|l|=1\end{array}$ & $\begin{array}{l}3 v_{8} \\
|l|=3\end{array}$ \\
\hline $\begin{array}{l}1 \\
1 \\
1 \\
1\end{array}$ & $\begin{array}{r}-1 \\
1 \\
0 \\
+1\end{array}$ & $\begin{array}{l}+ \\
- \\
+ \\
+\end{array}$ & $12912.05(-03)$ & $\begin{array}{l}12877.59(00) \\
12880.56(-13) \\
12880.56(-05) \\
12883.05(05)\end{array}$ & $\begin{array}{l}12920.04(-06) \\
12929.82(-02) \\
\frac{12930.11(-02)}{12940.48(-02)}\end{array}$ & $\begin{array}{l}12968.50(-07) \\
12968.50(-07) \\
\frac{12968.52(-08)}{12968.50(-07)}\end{array}$ & 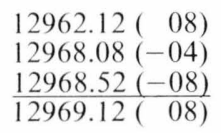 & $\begin{array}{l}12985.45(09) \\
13005.19(-10) \\
13005.61(-06) \\
13026.11(-06)\end{array}$ & $\begin{array}{l}13006.33(09) \\
13004.67(-17) \\
13005.61(-06) \\
13006.33(09)\end{array}$ \\
\hline $\begin{array}{l}2 \\
2 \\
2 \\
2 \\
2 \\
2\end{array}$ & $\begin{array}{r}-1 \\
2 \\
1 \\
0 \\
2 \\
+1\end{array}$ & $\begin{array}{l}+ \\
- \\
- \\
+ \\
+ \\
+\end{array}$ & $\begin{array}{l}19368.04(-05) \\
19368.50(03) \\
19368.92(-11) \\
19381.50(-02)\end{array}$ & 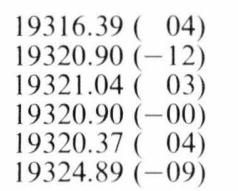 & $\begin{array}{l}19379.97(-15) \\
19394.39(16) \\
19394.81(04) \\
19395.23(-01) \\
19410.82(09)\end{array}$ & 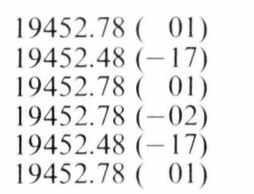 & 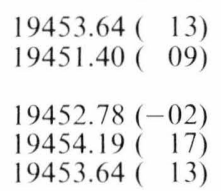 & 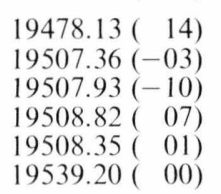 & 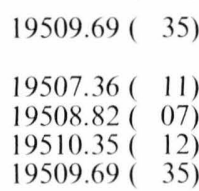 \\
\hline $\begin{array}{l}3 \\
3 \\
3 \\
3 \\
3 \\
3 \\
3 \\
3\end{array}$ & $\begin{array}{r}-1 \\
3 \\
2 \\
1 \\
0 \\
2 \\
3 \\
+1\end{array}$ & $\begin{array}{l}+ \\
- \\
- \\
- \\
+ \\
+ \\
+ \\
+\end{array}$ & $\begin{array}{l}25808.00(-05) \\
25822.51(-09) \\
25823.41(02) \\
25824.17(13) \\
25824.63(09) \\
25825.44(08) \\
25825.44(-01) \\
25841.95(-03)\end{array}$ & $\begin{array}{l}25755.03(-06) \\
25761.11(-09) \\
25761.57(25) \\
25761.57(26) \\
25761.03(-15) \\
25760.34(-03) \\
25759.87(03) \\
25766.61\left(\begin{array}{r}(02)\end{array}\right)\end{array}$ & 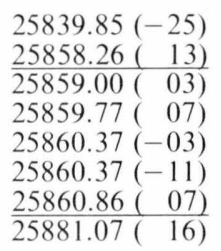 & 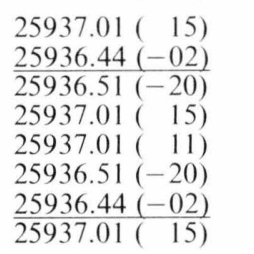 & $\begin{array}{l}25937.90(-01) \\
25933.83(06) \\
25934.91(-08) \\
25935.89(-20) \\
25937.01(11) \\
25938.58(06) \\
25939.28(03) \\
25937.90(-01)\end{array}$ & 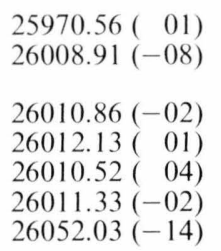 & $\begin{array}{l}26012.41(-03) \\
26006.40(12) \\
\\
26009.57(-08) \\
26012.13(01) \\
26013.53(-10) \\
26014.59(-07) \\
26012.41(-03)\end{array}$ \\
\hline & $\begin{array}{l}B_{0} \\
\alpha_{r} \\
\gamma_{r} \\
D_{J} \\
D_{J K} \\
\eta_{J} \\
q^{+} \\
Z_{\zeta_{1-}} \\
\zeta_{* *}\end{array}$ & & $\begin{aligned} 3222.506 & (0.004) \\
-5.585 & (0.004) \\
& \\
0.46 & (0.05) \\
8.34 & (0.12) \\
58.82 & (1.45) \\
4.241 & (0.010) \\
11970 & (8544) \\
-1.64 & (1.48) \\
0.79 & \end{aligned}$ & $\begin{aligned} & 3222.507(0.005) \\
& 2.350(0.005) \\
& \\
& 0.46 \quad(0.07) \\
& 8.33(0.17) \\
&-27.99(2.19) \\
& 1.438(0.012) \\
&-681(1191) \\
&+0.56(0.25) \\
& 0.57\end{aligned}$ & & $\begin{array}{r}3222.509(0.005) \\
-10.610(0.010) \\
-0.196(0.002) \\
0.50(0.07) \\
8.28(0.18) \\
57.74(0.61) \\
5.101(0.006) \\
-1335 \quad(68) \\
+0.672(0.025) \\
0.674\end{array}$ & & & \\
\hline
\end{tabular}

* $K$ and $l$ with same sign: + , otherwise:.$- \quad * *$ Calculated from force field

Underlined frequencies are center frequencies derived from quadrupole analysis. See Table 4. 
The most interesting parameter that has been determined is perhaps $Z$ (which was assumed to be independent of the other parameters in the fit) because it contains the Coriolis coupling constant $\zeta_{88}^{A}$, which can also be calculated from the force field derived in [1].

Using $A_{0}=5750 \pm 50 \mathrm{MHz}$ (from the $r_{\mathrm{a} r}$ structure [1]) and $B_{0}$, one obtains:

$$
\zeta_{88}^{A}=1-\frac{Z+B_{0}}{A_{0}}=0.672(0.025)
$$

(the error is one standard error including the estimated uncertainty in $A$ ) compared to the value from the normal coordinate analysis: $\zeta_{88}^{A}=0.674$.

\section{$v_{7}$-State (see Figure 1)}

The next higher vibration in $\mathrm{CF}_{3} \mathrm{NC}$ is $v_{7}$, the $\mathrm{CF}_{3}$ rocking mode, also of $E$-species, at $458 \mathrm{~cm}^{-1}$. In trifluoro acetonitrile this state appears to the low frequency side of the ground state - and this is also the case for the isocyanide. The population of the $v_{7}=1$ state is $11.8 \%$ (the $v_{7}=2$ state has a population of less than $1 \%$ and was not observed).

$\alpha_{7}$ and $q_{7}^{+}$are well determined, and in view of the quite precise evaluation of $\zeta_{88}^{A}$ and the good agreement with the value calculated from the force field, it was hoped that things would work equally well in the $v_{7}$ state. The accuracy of the $Z$-parameter, however, (using the limited amount of measured transitions in this state) was insufficient to more than establish that the Coriolis coupling constant calculated from the force field $\left(\zeta_{77}^{A}=0.57\right)$ is quite reasonable. Extended measurements are needed in order to improve the accuracy of the $\zeta_{77}^{A}$ determination.

The fit was done as for $v_{8}$, except that the $\gamma$ - and $Y$-dependence were left out. The results are collected in Table 2.

\section{$v_{6}$-State (see Figure 1)}

Even weaker than the $v_{7}$ band, the $v_{6}$ band $\left(\mathrm{CF}_{3}\right.$ deformation with a population of $4 \%$ ) is barely discernable to higher frequency of the ground state, the upper $l$-type doubling component almost coinciding with the lower $l$-type doubling component of the $v_{8}$-state. Stark components from the surrounding states often interfere destructively with this band, and values for $\eta_{J}$ and $Z$ are highly tentative.
The fit was done as for $v_{7}$ and the results collected in Table 2.

\section{$v_{4}$-State (see Figure 1)}

The lowest frequency non-degenerate state $v_{4}$ (Sym. $\mathrm{CF}_{3}$ deformation, population $3 \%$ ) appears in the highest resolution spectra to lower frequency from, but still on the flanks of the ground state bands. As in $v_{6}$, only a few transitions could be unequivocably assigned and the derived parameters are somewhat tentative. The fit has been carried out as for the ground state, using the Wilson-type symmetric top Hamiltonian. Measured frequencies are collected in Table 3 with the derived rotational constants.

Table 3. Measured Transitions (meas.-calc.) and derived Rotational Constants $(1 \sigma)$ for the $v_{4}$ State of $\mathrm{CF}_{3} \mathrm{NC}$ (MHz).

\begin{tabular}{llll}
\hline$J \backslash K$ & 0 & 1 & 3 \\
1 & $12889.34(-08)$ & $12889.34(-05)$ & \\
2 & $19334.12(02)$ & $19334.12(08)$ & \\
3 & $25778.72(00)$ & $25778.72(00)$ & $25778.10(00)$ \\
5 & $38667.74(00)$ & & $36668.81(00)$ \\
$B_{4}$ & $3222.362(0.006)$ & & \\
$\alpha_{4}$ & $0.147(0.006)$ & $\left(B_{0}=3222.509(0.002)\right)^{\mathrm{a}}$ \\
$D_{J}$ & $0.69(0.11)$ & & \\
$D_{J K}$ & $8.61(0.60)$ & \\
\hline
\end{tabular}

Centrifugal Distortion Constants in $\mathrm{kHz} .{ }^{\mathrm{a}}$ see [1].

Table 4. Quadrupole Hyperfine Structure in vibrationally excited States of $\mathrm{CF}_{3} \mathrm{NC}$.

\begin{tabular}{|c|c|c|c|c|}
\hline & & $v_{7}$ & $v_{8}$ & $2 v_{8}$ \\
\hline $\begin{array}{l}J: 1 \rightarrow 2 \\
K=0\end{array}$ & $\begin{array}{lll}F \rightarrow & F^{\prime} \\
1 & 1 & \\
1 & 2 / 2 & 3 \\
0 & 1 / 2 & 2\end{array}$ & $\begin{array}{l}12880.54 \\
12880.79\end{array}$ & $\begin{array}{l}12929.60 \\
12930.09 \\
12930.42\end{array}$ & $\begin{array}{l}12967.80 \\
12968.50 \\
12968.77\end{array}$ \\
\hline $\begin{array}{l}J: 2 \rightarrow 3 \\
K=2\end{array}$ & $\begin{array}{ll}1 & 2 \\
3 & 4 \\
2 & 3\end{array}$ & & $\begin{array}{l}19394.21 \\
19394.30 \\
19394.72\end{array}$ & \\
\hline $\begin{array}{l}J: 3 \rightarrow 4 \\
K=3\end{array}$ & $\begin{array}{ll}2 & 3 \\
4 & 5 \\
3 & 4\end{array}$ & $\begin{array}{l}25760.77 \\
25761.03^{\mathrm{a}}\end{array}$ & $\begin{array}{l}25858.20^{c} \\
25858.69\end{array}$ & $\begin{array}{l}25936.36^{b} \\
25936.51\end{array}$ \\
\hline & $\begin{array}{ll}4 & 5 \\
3 & 4\end{array}$ & & $\begin{array}{l}25860.80^{\mathrm{a}} \\
25861.24\end{array}$ & $\begin{array}{l}25939.20^{\mathrm{d}} \\
25939.70\end{array}$ \\
\hline
\end{tabular}

\footnotetext{
a $K$ and $l$ with same sign. $\quad$ c $K$ and $l$ with opposite sign.

b $l=0$.
} 


\section{Discussion}

Of major interest are the values of the $\zeta$-constants $\zeta_{66}^{A}, \zeta_{77}^{A}$, and $\zeta_{88}^{A}$, which can be obtained from the determined $(A \zeta)_{v}$-values, assuming $(A \zeta)_{v}=A_{v} \zeta_{v v}^{A}$ and estimating:

$$
A_{0}=A_{z}-1 / 2 \sum d_{i} \alpha_{i}(\text { harm }) .
$$

$A_{z}$ was determined in [1] using the harmonic contributions to $\alpha$, calculated from the force field. This force field also allows a calculation of the $\zeta_{v v}^{A}$ constants for the degenerate vibrations. These values are listed in Table 2 together with the experimentally determined ones. The accuracy of these determinations depends strongly on the selection of transitions, and thus on the intensity of the excited states, and it is not surprising that only the $v_{8}$ data provide a reliable determination of the Coriolis constant. The data for $v_{7}$ show that the value calculated from the force field is reasonable, whereas all that can really be established from the $v_{6}$ fit is

[1] Dines Christen, Klaus Ramme, Bernhard Haas, Heinz Oberhammer, and Dieter Lenz, J. Chem. Phys. 80, 4020 (1984).

[2] D. Lenz, J. Fluor. Chem. 24, 523 (1984).

[3] H. Bürger, private communication.

[4] G. Amat, H. H. Nielsen, and G. Tarrago, p. 261-267, Rotation-vibration of Polyatomic Molecules, Marcel Dekker Inc., New York 1971.

[5a] W. F. Edgell and R. M. Potter, J. Chem. Phys. 24, 80 (1956).
Microwave Spectra of Excited Vibrational States of $\mathrm{CF}_{3} \mathrm{NC}$

the sign of $\zeta_{66}^{A}$, which in this case is negative - in contrast to the $\zeta$ s for $v_{7}$ and $v_{8}$.

Using the numerical values of $\zeta_{66}^{A}$ and $\zeta_{77}^{A}$ from the force field (with the experimentally determined sign) and the experimental value for $\zeta_{88}^{A}$, one can calculate $\zeta_{55}^{A}$ from the zeta sum rule for $C_{3 v}$ molecules [9] (with $n$ number of axial atoms):

$$
\sum_{\mathrm{t}} \zeta_{\mathrm{tt}}^{A}=n-2+\frac{B_{0}}{2 A_{0}}=1.28 \quad \rightarrow \zeta_{55}^{A}=0.84
$$

and the value calculated from the force field is 0.83 .

Combinations of the $\zeta$ s using other signs are all in conflict with the force field calculation (in fact, most other calculations yield $\left.\left|\zeta_{55}^{A}\right|>1\right)$.

The data derived here should facilitate the study of high resolution vibrational, as well as $\mathrm{mm}$ rotational spectra, which will be needed to clear the assignment of $v_{2} / v_{5}$, although it must be noted that model calculations show the differences in the Coriolis coupling constants upon a change of $v_{2} / v_{5}$ assignment are very minute.

[5b] J. A. Faniran and H. F. Shurvell, Spectrochim. Acta 26, 1459 (1970).

[6] L. F. Thomas, J. S. Heeks, and J. Sheridan, Z. Elektrochem. 61,935 (1957).

[7] M. J. Whittle, J. G. Baker, and G. Corbelli, J. Mol. Spectry. 40, 388 (1971).

[8] A. P. Cox, D. J. Finnigan, and M. J. Whittle, J. Mol. Spectry. 49, 124 (1974).

[9] H. C. Allen and P. C. Cross, p. 59, Molecular Vibrotors, J. Wiley \& Sons Inc., New York 1963. 